\title{
PRELIMINARY STUDY ON THE IMPACT OF POLYMER-LIPID TYPES AND RATIO TO POLYMERIC-LIPID HYBRID NANOPARTICLE
}

\author{
OKTAVIA EKA PUSPITA, FERRI WIDODO, MONICA ANDIKA PUTRI, ISWA ROSSARIZA, AVIOLA FADHILLA, NI \\ PUTU JUNITA SARI
}

Department of Pharmacy, Faculty of Medicine, Brawijaya University, Malang, Indonesia 65145

Email: oktaviaeka@ub.ac.id

Received: 01 Apr 2021, Revised and Accepted: 11 May 2021

\section{ABSTRACT}

Objective: This study aims to determine the best lipid to polymer ratios in polymeric-lipid nanoparticles using various types and ratios of polymers and lipids.

Method: Polymeric-lipid nanoparticle was prepared using the modified one-step nanoprecipitation method. This study used chitosan and Na alginate as polymers and lecithin and egg phosphatidylcholine as lipids. The lipid was crossed-combined with polymer in various ratios, i. e $12.5 \%$, $25.0 \%$, and $37.5 \%$. On its preparation, Cinnamomum burmanii extract was loaded into the resulted polymeric-lipid nanoparticle as an active substance model. The results were assessed its particle surface characteristics including particle size, polydispersity index, and zeta potential.

Results: Twelve formulas resulted from crossed-combination between the lipid and polymer were used in this study. Polymeric-lipid nanoparticles resulted from the combination of egg phosphatidylcholine/Na alginate has particle size, polydispersity index, and zeta potential of $380.07 \pm 3.52 \mathrm{~nm}$, $0.66 \pm 002$, and-30.6 $\pm 1.15 \mathrm{mV}$, respectively.

Conclusions: The best lipid to polymer ratio and type was observed in egg phosphatidylcholine: Na alginate. The particle surface characteristics were better compared to other combinations.

Keywords: Polymeric-lipid hybrid nanoparticle, Egg phosphatidylcholine, Lecithin, Na alginate, Chitosan

(C) 2021 The Authors. Published by Innovare Academic Sciences Pvt Ltd. This is an open access article under the CC BY license (https://creativecommons.org/licenses/by/4.0/) DOI: https://dx.doi.org/10.22159/ijap.2021v13i4.41663. Journal homepage: https://innovareacademics.in/journals/index.php/ijap

\section{INTRODUCTION}

Nanotechnology has been applied in various materials to develop nanoparticles drug delivery systems because its nanoscale characteristics are capable of altering the properties of a drug, including its bioavailability, biodistribution, and pharmacokinetics. One of the nanoparticle carriers used to deliver active substances is a polymeric-lipid nanoparticle. This nanoparticle conceptually a combination of polymeric nanoparticles and liposome. The combination is due to various restrictions of liposomes and biodegradable polymeric nanoparticles. The polymeric-lipid nanoparticle is a novel drug delivery system that has more benefits as compared to liposomes and nanoparticles [1-3]. Liposomes are spherical vesicles with a lipoidal bilayer membrane containing amphiphilic lipid molecules which are used to deliver both hydrophobic and hydrophilic drugs that protect the drug from the external environment $[1,3,4-6]$. But the application of liposomes in drug delivery was limited because of the low encapsulation efficiency of low water-soluble drugs such that the release of entrapped the drug occurs quickly resulting in the instability of the drug. While polymeric nanoparticles are typically hydrophobic which leads to short in vivo circulation time resulting in their low bioavailability [7-9]. Addressing the limitations of polymeric nanoparticles and liposomes, a new generation delivery vehicle of therapeutics termed polymer-lipid hybrid nanoparticles (or polymeric-lipid nanoparticles, PLNs) has been developed. The PLNs, which combine the characteristics of both polymeric nanoparticles and liposomes, comprises three components, i. e a polymer core in which the therapeutic substances are encapsulated, an inner lipid layer enveloping the polymer core, and an outer lipid-PEG layer. Instead of a three-layer, PLNs can also be prepared without the PEG layer but only lipid (usually phospholipid) and polymer [10].

One of the preparation methods for PLNs preparation is one-step nanoprecipitation. The modification of this method from the previous study is used in this study (explained in section Methods) [1,11-14]. The polymer and the active substance to-be-encapsulated are dissolved in water-miscible organic solvent then dispersed into water containing dispersed lipid. Once the nanoparticle is formed simultaneously the lipid self-assemble on the surface of the nanoparticles. The self-assembly mechanism is based on hydrophobic interactions. Hydrophobic of lipids is attached to the polymer nanoparticle surface and the hydrophilic heads to the external aqueous surrounding resulting in the formation of PLNs that are stabilized by the lipid [1].

Physical characteristics of PLNs are determined by different combination types of polymer and lipid. Those may result in various particle sizes, zeta potential, as well as its active substance loading capacity [15]. Particle size is a crucial factor to determine the absorption, distribution, and in vivo performance of nanoparticles. In general, nanoparticles have a higher cellular uptake efficiency than microparticles. A decrease of the particle size to $<1 \mathrm{~mm}$ increases particle cellular uptake efficiency. Nanoparticles with a particle size $<100 \mathrm{~nm}$ are efficiently taken up in Peyer's patches, and then absorbed into the systemic circulation [11]. This research aimed to find the best combination of natural biodegradable polymer and lipid in developing polymeric lipid nanoparticles to encapsulate low stability and bioavailability substance as contained in Cinnamomum burmanii extract when administered by the oral route. The PLNs development for Cinnamomum burmanii extract is expected to be a prospectus formula for further study on its efficacy on the animal model. Nanotechnology applied to herbal delivery has a profound impact on its permeability and bioavailability [16].

\section{MATERIALS AND METHODS}

\section{Materials}

Medium molecular weight chitosan (Sigma Aldrich Singapore), egg phosphatidylcholine (Sigma Aldrich Singapore), sodium alginate, soybean lecithin, acetone, methanol, purified water, and Poloxamer 407 were purchased from Duta Jaya Laboratory (Indonesia), Cinnamomum burmanii dried bark extract purchased from PT. Borobudur (Indonesia).

\section{Methods}

Polymeric lipid nanoparticle preparation

Polymeric lipid nanoparticle was prepared using the modified onestep nanoprecipitation method adopted from the previously 
explained method (fig. 1) [1, 11-14]. A list of lipid to polymer variations is presented in table 1 . Chitosan was dissolved in purified water and the $\mathrm{pH}$ was adjusted to $2-4$ using $\mathrm{HCl} 0.1 \mathrm{~N}$ solution while stirring using a magnetic stirrer. While in a separated container each soybean lecithin and $C$. burmanii extract was dissolved in acetone and methanol respectively. The three solutions were then combined in one container and called the organic phase. In another beaker glass, the aqueous phase was prepared by dissolving Poloxamer 407 and water. The organic phase then added gradually into the aqueous phase while stirred using IKA Overhead stirrer. The ratio of organic phase to aqueous phase was $1: 10(\mathrm{v} / \mathrm{v})$. Once the organic phase was completed added into the aqueous phase the mixing was continued using IKA Ultra Turrax at $8.000 \mathrm{rpm}$ for $5 \mathrm{~min}$. The mixing then continued using IKA Overhead stirrer for $24 \mathrm{~h}$ at room temperature to evaporate the organic phase. The resulted dispersion was then centrifuged at $5000 \mathrm{rpm}$ for $10 \mathrm{~min}$ to separate large aggregate and the supernatant is collected and carried out for second centrifugation at $10.000 \mathrm{rpm}$ for $5 \mathrm{~min}$. The final supernatant was collected which contained polymeric-lipid nanoparticle cinnamon extract. A slight difference procedure was carried out to prepare polymeric-lipid nanoparticles using polymer $\mathrm{Na}$ alginate. The organic phase consisted of egg phosphatidylcholine, Na alginate, and cinnamon extract which dissolved directly into acetone-methanol $(3: 2 \mathrm{v} / \mathrm{v})$. The next step was similar to previous procedures.
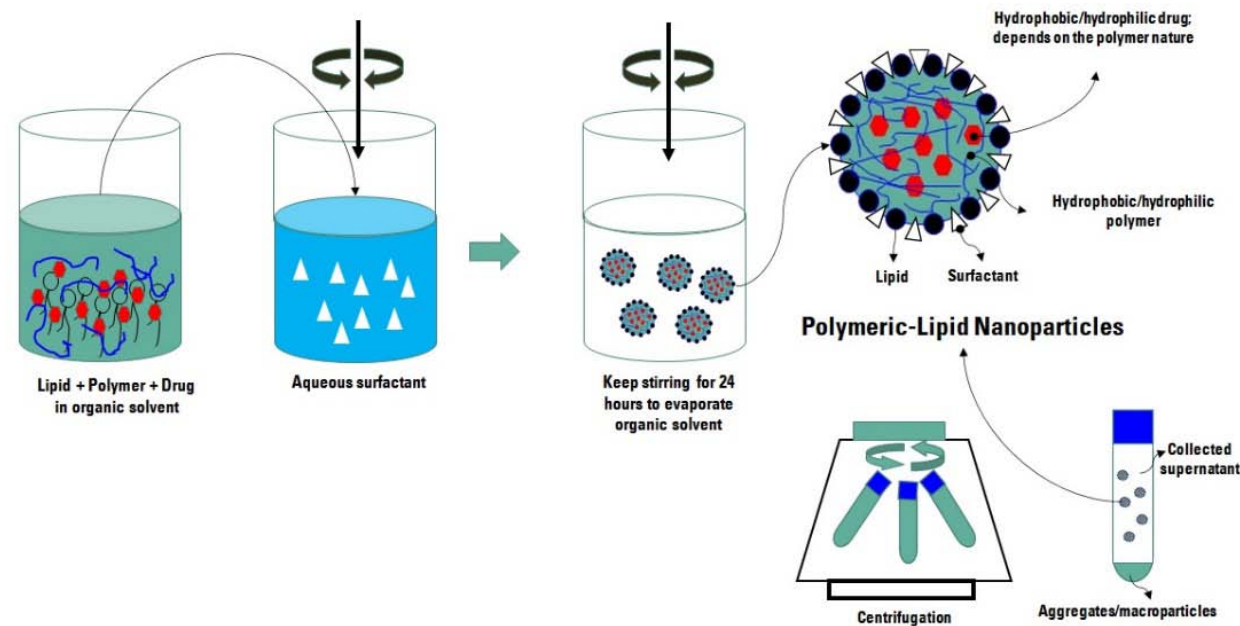

Fig. 1: Modified one-step method forming polymeric-lipid nanoparticles: lipid, polymer, and drug is dissolved in organic solvents, can be methanol combined with acetone in a certain proportion. This solution is an organic phase which added into an aqueous phase containing surfactant solution. The solution is added while homogenizing in optimizing nanoparticle formation besides the self-assembled process. The polymeric-lipid nanoparticle is separated from the large aggregate by centrifugation

\section{Particle size analysis}

Polymeric-lipid nanoparticle particle size, polydispersity index, and zeta potential were measured using Dynamic Light Scattering (DLS) method (Malvern Instrumen Zetasizer ZS). In this preliminary research of polymeric-lipid nanoparticle physical characteristic was assessed using those data. This is in considering that nanoparticle surface characteristics have a profound impact on overall nanoparticles performance. Therefore in this preliminary stage, the best lipid to polymer ratio and type were determined based on those data.

Table 1: Lipid to polymer type and ratio

\begin{tabular}{|c|c|c|c|c|c|c|c|c|c|}
\hline Formulation 1 & & F1.1 & F1.2 & F1.3 & Formulation 3 & & F3.1 & F3.2 & F3.3 \\
\hline \multirow[t]{4}{*}{ Organic phase } & Cinnamon Extract (mg) & 200 & 200 & 200 & Organic phase & Cinnamon Extract (mg) & 200 & 200 & 200 \\
\hline & Lecithin (mg) & 16 & 32 & 48 & & Lecithin (mg) & 16 & 32 & 48 \\
\hline & Na alginate $(\mathrm{mg})$ & 112 & 96 & 80 & & Chitosan (mg) & 112 & 96 & 80 \\
\hline & Acetone-metanol (v/v) & $3: 2$ & $3: 2$ & $3: 2$ & & Acetone-metanol (v/v) & $3: 2$ & $3: 2$ & $3: 2$ \\
\hline Aqueous phase & Poloxamer 407 (\%) & 1 & 1 & 1 & Aqueous phase & Poloxamer 407 (\%) & 1 & 1 & 1 \\
\hline Formulation 2 & & F2.1 & F2.2 & F2.3 & Formulation 4 & & F4.1 & F4.2 & F4.3 \\
\hline \multirow{4}{*}{ Organic phase } & Cinnamon Extract (mg) & 200 & 200 & 200 & Organic phase & Cinnamon Extract (mg) & 200 & 200 & 200 \\
\hline & Egg PC (mg) & 16 & 32 & 48 & & Egg PC (mg) & 16 & 32 & 48 \\
\hline & Na alginate $(\mathrm{mg})$ & 112 & 96 & 80 & & Chitosan (mg) & 112 & 96 & 80 \\
\hline & Acetone-metanol (v/v) & $3: 2$ & $3: 2$ & $3: 2$ & & Acetone-metanol (v/v) & $3: 2$ & $3: 2$ & $3: 2$ \\
\hline Aqueous phase & Poloxamer 407 (\%) & 1 & 1 & 1 & Aqueous phase & Poloxamer 407 (\%) & 1 & 1 & 1 \\
\hline
\end{tabular}

Notes: Formulation 1 uses soy lecithin as lipid and Na alginate as polymer. The lipid to polymer combination is varied in three ratios, i. e F1.1 is $12.5 \%$, F1.2 is $25.0 \%$, and F1.3 is 37.5\%. Likewise, formulation 2, 3, and 4 have similar ratios of lipid to polymer but uses a different type of lipid and polymer.

\section{RESULTS AND DISCUSSION}

As observed in many studies that polymeric-lipid nanoparticle has the advantages combination of liposome and polymer nanoparticle, therefore it has the potential to increase absorption through the digestive tract and the stability of compounds in the systemic circulation [17-19]. In this regard, this research used Cinnamomum burmanii extract as a model for the active substance loaded into the nanoparticles. The Cinnamon extract has been studied on animals and clinical trials and showed potential benefits for diabetic type 2 conditions [20]. The preparation of PLNs containing Cinnamon extracts was using the one-step nanoprecipitation method. The polymer nanoparticles are self-assembled and the lipid molecules are conjugated. In this study, the polymer precipitates to form a 
hydrophilic core to encapsulate the Cinnamon extract. Lecithin or egg phosphatidylcholine conjugate self-assemble around the polymer core to form a lipid layer. This study did not involve PEGylation.

In table 1, there are twelve formula variations with the difference between formula is on the type of lipids and polymers combination and ratio. Within each type formula variant, there are three formulas that the lipid to polymer ratios are 1:7, 1:3, and 3:5 (12.5\%, 25.0\%, $37.5 \%$ respectively). The distinguishing variable between the variations of the formula is the type of lipid and polymer. This variation is to find which type of combination is better. In consideration of chitosan has the advantage in improving cell uptake and bioavailability of the poorly soluble drug [21], it is chosen in this research and compared to another polymer $\mathrm{Na}$ alginate. Both of them are biodegradable polymers.
The results of the various types of lipid and polymer combinations are described in table 2 . The measured impact of the lipid and polymer type is based on the PLNs surface physical characteristics including particle size, polydispersity index, and zeta potential. The most favorable combination is observed in the lecithin/Na alginate combination at a ratio of 3:5 (F1.3) respectively. Overall, looks like PLNs produced with chitosan resulting in a larger particle size amongst all. When lipid is kept the same, PLNs produced with chitosan has a larger particle size compared to $\mathrm{Na}$ alginate. On the other hand, when the polymer is kept the same, PLNs produced with egg phosphatidylcholine have a smaller particle size compared to lecithin. Chitosan is a natural cationic copolymer and has hydrophilic nature while alginate is a natural polysaccharide and negatively charge polymer $[21,22]$. Therefore, the resulting core polymer nanoparticles in these PLNs are hydrophilic.

Table 2. Polymeric lipid nanoparticle surface characteristics

\begin{tabular}{|c|c|c|c|c|c|c|}
\hline & \multicolumn{3}{|c|}{ Formulation 1: Lecithin/Na Alginate } & \multicolumn{3}{|c|}{ Formulation 3: Lecithin/Chitosan } \\
\hline & F1.1 & F1.2 & F1.3 & F3.1 & F3.2 & F3.3 \\
\hline Particle size (nm) & $501,93 \pm 5,50$ & $465,87 \pm 5,54$ & $380,07 \pm 3,52$ & $1317 \pm 6,93$ & $1047,33 \pm 6,11$ & $960,17 \pm 4,24$ \\
\hline Polidispersity index & $0,91 \pm 0,02$ & $0,83 \pm 0,14$ & $0,66 \pm 0,02$ & $0,92 \pm 0,09$ & $0,68 \pm 0,03$ & $0,65 \pm 0,01$ \\
\hline \multirow[t]{3}{*}{ Zeta potensial $(\mathrm{mV})$} & $-30,53 \pm 3,20$ & $-31,4 \pm 1,91$ & $-30,6 \pm 1,15$ & $41,8 \pm 0,26$ & $45,63 \pm 0,81$ & $34,93 \pm 0,84$ \\
\hline & \multicolumn{3}{|c|}{ Formulation 2: Egg Phosphatidylcholine/Na Alginate } & \multicolumn{3}{|c|}{ Formulation 4: Egg Phosphatidylcholine/Chitosan } \\
\hline & F2.1 & F2.2 & F2.3 & F4.1 & F4.2 & F4.3 \\
\hline Particle size (nm) & $438,63 \pm 6,70$ & $478,26 \pm 4,80$ & $372,36 \pm 3,62$ & $1164,33 \pm 24,95$ & $854,967 \pm 1,66$ & $748,967 \pm 5,23$ \\
\hline Polidispersity index & $0,64 \pm 0,01$ & $0,59 \pm 0,04$ & $0,83 \pm 0,05$ & $0,741 \pm 0,024$ & $0,559 \pm 0,011$ & $0,572 \pm 0,048$ \\
\hline Zeta potensial $(\mathrm{mV})$ & $-25,13 \pm 1,53$ & $-24,63 \pm 0,98$ & $-25,76 \pm 3,88$ & $43,933 \pm 1,159$ & $42,567 \pm 0,351$ & $41 \pm 0,458$ \\
\hline
\end{tabular}

Notes: Data represents mean \pm SD $(n=3)$

The important formulation components that contribute to PLNs characteristics is the lipid to polymer mass ratio. The lipid has to be sufficiently covers the surface of the polymer particle $(1,4)$. The role of the lipid coating layer is influencing the particle size [15]. This research observed that the higher the lipid mass ratio to polymer, the smaller the particle size. This trend in particle size is observed in all formula combinations. This may be an indication of the number of lipids is in the range to cover the entire surface of the polymer nanoparticle core. The highest ratio of the lipid to polymer in this study is $37.5 \%$. On the other hand, increasing the polymer concentration resulted in larger PLNs [23]. This corresponds to the results within every formulation variation described in table 2 . The higher the ratio of the polymer to lipid resulting in larger PLNs' particle size.

As stated in the section Introduction, the PLNs preparation method applied in this study is a modification from the one-step nanoprecipitation method. The preparation involving surfactant in helping nanoparticle formation by dispersing water-immiscible organic solvent into aqueous surfactant solution. This will initiate globule formation and be enhanced by high-speed homogenization. This process is similar to emulsification preparation. The surfactant looks like contributes to the PLNs particle size. We did a trial by changing surfactant type in formulation F3.3 (lecithin-chitosan) which the surfactant is Poloxamer 407 . When it changed to Tween 80 , the particle size drastically changed to $289.33 \pm 10.3 \mathrm{~nm}$ (previously $960.17 \pm 4.24 \mathrm{~nm}$ ). It indicates that using this preparation method, the type of surfactant is matters. Nevertheless, this still needs further study on the impact of surfactant on the physical characteristic of PLNs.

\section{CONCLUSION}

This preliminary study has observed that the most favorable polymeric-lipid nanoparticles are using lecithin/ $\mathrm{Na}$ alginate in a ratio of $37.5 \%$. The PLNs core is a hydrophilic polymeric core considering the chitosan and $\mathrm{Na}$ alginate are hydrophilic. This finding needs further study on the active substance loading capacity, stability, and shell morphology.

\section{ACKNOWLEDGMENT}

The authors are grateful Faculty of Medicine, Brawijaya University for funding this work.

\section{FUNDING}

This work was supported by the Faculty of Medicine, Brawijaya University, Indonesia (grant number: 25/SK/UN10. F08.06/KS/2018).

\section{AUTHORS CONTRIBUTIONS}

Oktavia Eka Puspita generated research plan, conceptualization, methodology, formal analysis, and writing original draft preparation and revision. Ferri Widodo performed formal analysis. Monica Andika Putri, Iswa Rossariza, Aviola Fadhilla, Ni Putu Junita Sari performed in data collection.

\section{CONFLICTS OF INTERESTS}

All authors declare no conflict of interest.

\section{REFERENCES}

1. Zhang L, Zhang L. Lipid-polymer hybrid nanoparticles: synthesis, characterization and application. Nano Life 2010;1:73-163.

2. Zhang L, Chan JM, Gu FX, Rhee J, Wang AZ, Radovic Moreno AF et al. Self-assembled lipidpolymer hybrid nanoparticles a robust drug delivery platform. ACS Nano 2008;2:702-1696.

3. Enlow EM, Luft JC, Napier ME, Desimone JM. Potent engineered PLGA nanoparticles by virtue of exceptionally high chemotherapeutic loadings. Nano Lett 2011;11:13-808.

4. Albanese A, Tang PS, Chan WCW. The effect of nanoparticle size, shape, and surface chemistry on biological systems. Annu Rev Biomed Eng 2012;14:1-16.

5. Sharma A, Sharma US. Liposomes in drug delivery: Progress and limitations. Int J Pharm 1997;154:40-123.

6. Bonde S, Nair S. Advances in liposomal drug delivery system: Fascinating types and potential applications. Int J Appl Pharm 2017;9:1-7.

7. Otsuka H, Nagasaki Y, Kataoka K. PEGylated nanoparticles for biological and pharmaceutical applications. Adv Drug Delivery Rev 2003;55:19-403.

8. Sheng Y, Liu C, Yuan Y, Tao X, Yang F, Shan X, et al. long-circulating polymeric nanoparticles bearing a combinatorial coating of PEG and water-soluble chitosan. Biomaterials 2009;30:2340.

9. Kandel PK, Fernando LP, Ackroyd PC, Christensen KA. Incorporating functionalized polyethylene glycol lipids into 
reprecipitated conjugated polymer nanoparticles for bioconjugation and targeted labeling of cells. Nanoscale 2011;3:45-1037.

10. Zhang L, Chan JM, Gu FX, Rhee JW, Wang AZ, Radovic Moreno AF, et al. Self-assembled lipid-polymer hybrid nanoparticles: a robust drug delivery platform. ACS Nano 2008;2:702-1696.

11. Panyam J, Labhasetwar V. B iodegradable nanoparticles for drug and gene delivery to cells and tissue. Adv Drug Delivery Rev 2003;55:47-329.

12. Govender T, Stolnik S, Garnett MC, Illum L, Davis SS. PLGA nanoparticles prepared by nanoprecipitation: drug loading and release studies of a water-soluble drug. J Controlled Release 1999;57:171-85.

13. Dinesh KV, Verma PRP, Singh SK. Development and evaluation of biodegradable polymeric nanoparticles for the effective delivery of quercetin using a quality by design approach. Food Sci Technol 2015;61:8-330.

14. Yu K, Zhao J, Yu C, Sun F, Liu Y, Zhang Y, et al. Role of four different kinds of polyethyleneimine (PEIs) in preparation of polymeric lipid nanoparticles and their anticancer activity study. J Cancer 2016;7:82-872.
15. Hadinoto K, Sundaresan A, Cheow WS. Lipid-polymer hybrid nanoparticles as a new generation therapeutic delivery platform: a review. Eur J Pharm Biopharm 2013;85:43-427.

16. Sivapriya V, Ponnarmadha S, Azeezand NA, Sudarshanadeepa V. Novel nanocarriers for ethnopharmacological formulations. Int J Appl Pharm 2018;10:26-30.

17. Torchilin VP. Recent advances with liposomes as pharmaceutical carriers. Nat Rev Drug Discovery 2005;4:60-145.

18. Maurer N, Fenske DB, Cullis PR. Developments in liposomal drug delivery systems. Expert Opin Biol Ther 2001;1:47-923.

19. Yang XZ, Dou S, Wang YC, Long HY, Xiong MH, Mao CQ, et al. Singlestep assembly of cationic lipid-polymer hybrid nanoparticles for systemic delivery of siRNA. ACS Nano 2012;6:65-4955.

20. Anderson RA. Chromium and polyphenols from cinnamon improve insulin sensitivity. Proc Nutr Soc 2008;67:48-53.

21. Li J, Cai C, Sun T, Wang L, Wu H, Yu G. Chitosan-based nanomaterials for drug delivery. Molecules 2018;23:1-26.

22. Ahmadi F, Oveisi Z, Samani SM, Amoozgar Z. Chitosan-based hydrogels: characteristics and pharmaceutical applications. Res Pharm Sci 2015;10:1-16.

23. Chan JM, Zhang L, Yuet KP, Liao G, Rhee JW, Langer R, et al. PLGA-lecithin-PEG core-shell nanoparticles for controlled drug delivery. Biomaterials 2009;30:34-1627. 